\title{
Objective Evaluation Method of Fusion Image Quality Based on the Completed Local Binary Pattern
}

\author{
Jing $\mathrm{Xu}^{1, *}$ and Lin Cheng ${ }^{2}$
}

${ }^{I}$ School of Electronic Engineering, Xi'an University of Posts and Telecommunications, Xi'an 710121, China

${ }^{2}$ Department of Electrical Engineering, Wayne State University, Detroit, MI 48202, United States

Received 9 November 2017; Accepted 24 May 2018

\begin{abstract}
Image fusion refers to extracting information from multiple images on the same scene to acquire accurate and reliable image description of the same scene. Multiple image fusion models and algorithms have emerged in recent years. Hence, effectively evaluating fusion image quality has become a problem that requires urgent solution. An existing objective evaluation method of fusion image quality has disregarded the influence of quality comparison between original images on fusion effect, and the evaluation result is inconsistent with human subjective feeling. An objective evaluation algorithm of fusion image quality was proposed in this study to reveal the influence of quality comparison between original images on fusion effect. A completed local binary pattern (CLBP) was used to construct a CLBP operator. A CLBP texture detection of original images for fusion was performed. Qualities of the parts of the original images for fusion were compared. The proportion of the favorable quality parts of images to be fused and transferred to fusion result was defined as an objective evaluation index of the fusion effect. Finally, a comparative verification of the objective evaluation algorithm proposed in this study was conducted by using the edge-based and feature mutual information (FMI) algorithms. Results show that a consistency proportion of the CLBP-based evaluation algorithm with subjective evaluation is $80 \%$ when several mainstream fusion algorithms are used to fuse the identical original images. Compared with the edge-based and FMI algorithms, the consistency proportion of the proposed algorithm with subjective evaluation is increased by $20 \%$ and $40 \%$ respectively. The consistency proportion of the CLBP-based algorithm with subjective valuation is $75 \%$ when a contrast algorithm is used to fuse different original images. Relative to the edge-based and FMI algorithms, the consistency proportion of the proposed algorithm with subjective evaluation is increased by $25 \%$ and $50 \%$ respectively. This study provides a reference for selecting a fusion algorithm.
\end{abstract}

Keywords: Image fusion, Completed local binary pattern, Quality comparison, Objective evaluation

\section{Introduction}

Image fusion can generate a high-quality image through certain processing of multiple images on the same scene. Image fusion technology has been widely applied to various fields, such as medical imaging, object identification, satellite remote sensing, weather forecast, and transportation. Image fusion algorithm can be largely classified into two types, namely, spatial domain-based image fusion [1-2] and transform domain-based image fusion [3-4]. A reasonable evaluation method of fusion image quality is crucial to selectively comparing fusion algorithms and designing new fusion algorithms. Therefore, establishing a universal fusion image quality evaluation criterion is a present problem that urgently requires a solution.

Currently, existing equation methods of fusion image quality can be divided into subjective and objective methods. An evaluation process of the subjective evaluation method is tedious and is affected by human psychological factors with poor stability. An evaluation result of the objective evaluation method is unique, thereby considering it the preferred evaluation method. The objective evaluation

*E-mail address: codeidler@163.com

ISSN: $1791-2377$ @ 2018 Eastern Macedonia and Thrace Institute of Technology. All rights reserved. doi:10.25103/jestr.113.15 method can be classified into two types, namely, supervised evaluation methods [5] and unsupervised evaluation methods [6-20] in accordance with their different working principles. Several parameters are used in the unsupervised evaluation method to conduct a quantitative evaluation of the fusion result. This method does not need to acquire a standard image during the evaluation process, thus contributing to extensive application. Furthermore, the supervised evaluation method requires a standard image during the evaluation process. The two evaluation methods have disregarded the influence of quality comparison between images for fusion on fusion image quality. Consequently, the evaluation result is inconsistent with the actual situation under a few circumstances or the quality measurement of images for fusion is insufficiently accurate; hence, the quality evaluation of a fusion image has poor consistency with human subjective feeling. Therefore, combining image qualities for fusion to realize the accurate evaluation of fusion image quality requires an urgent solution.

Therefore, an advantage of a completed local binary pattern (CLBP) in measuring textural features was considered in this study to conduct a quantitative description of image qualities for fusion, and an unsupervised multifocus fusion image quality evaluation method that combines image qualities for fusion was proposed. This method with 
simple implementation demonstrates favorable consistency with the subjective feeling in the evaluation result. Moreover, this study can provide a reference for selecting fusion algorithms.

\section{State of the art}

Objective evaluation methods conduct a quantitative description of image quality by measuring relevant indexes and are expected to reach a consistent perceiving effect with human visual system for image quality. In accordance with their different working principles, objective evaluation methods can be classified into two types, namely, supervised and unsupervised evaluation methods. Scholars have performed numerous studies on unsupervised evaluation methods. Haghighat et al. [6] proposed a fast feature mutual informationFMI algorithm to reduce the complexity of the FMI algorithm and the requirement for a high storage capacity; however, mutual information was used to describe the relationship between the original and the fusion images, which weakens the influence of image textural features on evaluation result. Pang et al. [7] used a quaternion-valued edge template and color image to conduct a quaternion convolution operation to acquire detailed information of color images and then presented the objective evaluation result of fusion image quality through numerous quantitative calculations. Pang method was mainly conducted for modeling of a color image using quaternion, but classical gradient operator was adopted for the template that is used to acquire detailed image information; and only horizontal and vertical edges were detected. Zhang et al. [8] compared the structural similarities between the image for fusion and the fusion image and used phase consistency images of the images for fusion to realize the weighing of structural similarity and evaluate the fusion image quality; however, the images for fusion were not compared. Luo et al. [9] disregarded the influence of the local details of images for fusion on the evaluation result when using principal component analysis method to select standard images. Zhou et al. [10] regarded single indexes used for the objective evaluation of an image fusion effect as a few properties used to describe the image fusion effect and considered image fusion evaluation as a multi-property decision-making problem, thereby failing to solve the contradiction difference between the evaluation indexes of the image fusion effect. Zhu et al. [11] used kernel Fisher to classify image fusion effects, but evaluation effects that belong to the same category cannot be compared. Wang et al. [12] used an information entropy ratio of a fusion image to relevant original image in the corresponding region to characterize the similarities between fusion and original images in this region regardless of image textural features. Wu et al. [13] established spectral and spatial information indexes to quantitatively evaluate fusion effect but disregarded the influence of the original image quality on fusion image quality. Hassen et al. [14] proposed using image contrast, sharpness, and structural preservation to evaluate fusion image quality, but image neighborhood deviation used to describe image textures was insufficiently elaborate. Petrović et al. [15] adopted a gradient information preservation of the original image in the fusion image to evaluate fusion effect, but the gradient used to describe an image texture was insufficiently elaborate. Omar et al. [16] utilized a gray-level co-occurrence matrix to express the preservation degree of textural details of the original image in the fusion image but disregarded the influence of the original image quality on the fusion effect. Hossny et al. [17] used localized mutual information to compare the similarities between the original and fusion image blocks regardless of the influence of the image textural features on the evaluation effect. Cvejic et al. [18] adopted mutual information on the basis of wavelet transform to measure the similarities between original and fusion image regions and used multiple factors that influence human visual feeling but did not compare the image qualities for fusion. Cvejic et al. [19] used mutual information to describe the relationship between the original and fusion images regardless of the influence of image textural features on the evaluation result. Blasch et al. [20] evaluated fusion effect by estimating an expression degree of important information in the original image by fusion image but did not compare the image qualities for fusion.

The results of the preceding study have mainly investigated the relationship between the image for fusion and the fusion image. However, few studies on a quality comparison between images for fusion and its influence on fusion effect are available. The advantage of a local binary pattern (LBP) in describing textural features was used in this study. Moreover, an improved LBP algorithm called CLBP was selected, and a CLBP operator named SMC was obtained by fusing three local textual descriptors (i.e., CLBP_S, CLBP_M, and CLBP_C) to describe the textural feature of each pixel. The $\bar{S} \bar{M} C$ operator was used to compare the image qualities for fusion. The fusion effect is improved when additional textures obtained from original images with favorable quality demonstrate an enhanced quality in the fusion result. The method not only considered the relationship between the image for fusion and fusion image but also compared image qualities for fusion; therefore, the obtained evaluation result was consistent with human subjective feeling.

The remainder of this study is organized as follows. Section 3 describes the construction of the SMC operator and design of the CLBP-based fusion image quality evaluation method. Section 4 consists of the experimental design and results analysis. Section 5 summarizes the conclusions.

\section{Methodology}

The same algorithm has different performances in fusing different types of images during the evaluation of fusion image quality. Moreover, the performances of different fusion algorithms may vary in fusing the same original images. This finding indicates that the original image quality has a certain influence on fusion image quality. Therefore, proposing a novel evaluation method of fusion image quality combined with the original image quality is necessary.

\subsection{Basic idea of the CLBP-based fusion image quality evaluation method}

Ojala et al. [21] proposed the LBP algorithm in 1996 to describe textural feature information. The LBP algorithm is theoretically simple and exhibits a minimal computational complexity. The LBP algorithm can describe local textural features of the image well, thereby acquiring a considerable concern from scholars in various countries [22-23]. In the past 20 years, the CLBP [24] algorithm has become comprehensive and elaborate in the local textural description. 
Therefore, the CLBP algorithm was used in this study to conduct a quantitative description.

The basic idea of the CLBP-based fusion image quality evaluation method is as follows: the original image for fusion is placed under the CLBP texture detection, and a proportion of the part with favorable quality transferred to fusion result is then defined as an evaluation index of the fusion effect.

\subsection{CLBP}

Three local textural descriptors, namely, window gray difference descriptor (CLBP-Sign, CLBP_S), window gradient different descriptor (CLBP-Magnitude, CLBP_M), and central pixel descriptor (CLBP-Center, CLBP_C), are proposed in the CLBP [24] algorithm to express the texture. Its computational formula is as follows:

$$
\begin{aligned}
& C L B P_{-} S_{P, R}\left(g_{c}\right)=\sum_{p=0}^{P-1} s\left(g_{p}-g_{c}\right) 2^{p}, s(x)=\left\{\begin{array}{cc}
1, & x \geq 0 \\
-1, & x<0
\end{array}\right. \\
& C L B P_{-} M_{P, R}\left(g_{c}\right)=\sum_{p=0}^{P-1} t\left(m_{p}, c\right) 2^{p} \\
& C L B P_{-} C_{P, R}\left(g_{c}\right)=t\left(g_{c}, c_{I}\right) \\
& t(x, c)=\left\{\begin{array}{l}
1, x \geq c \\
0, x<c
\end{array}\right. \\
& m_{p}=\left|g_{p}-g_{c}\right| \\
& c=\frac{1}{N} \sum_{n=0}^{N-1} \frac{1}{P} \sum_{p=0}^{P-1} m_{p}
\end{aligned}
$$

where $g_{p}$ is the gray level of pixel $\mathrm{p}$ in a neighborhood, $g_{c}$ is the gray value of the central pixel, $P$ is the number of pixels in the neighborhood, $R$ is the neighborhood radius, $N$ is the number of sub-windows divided by the image, and $c_{I}$ is the average value of the gray levels of the entire image. The formula is expressed as follows: $C L B P S_{P, R}$ refers to the original LBP; $C L B P \_M_{P, R}$ describes the gradient different information of the window, which had an important influence on the experimental result; $C L B P_{-} C_{P, R}$ reflects gray information of the central pixel in the window. Compared with the traditional LBP and its variants, combining the three descriptors, namely, CLBP_S, $C L B P_{-} M$, and $C L B P_{-} C$, can elaborately describe the texture.

\subsection{Description of the CLBP-based fusion image quality evaluation method}

The three descriptors, namely $C L B P S, C L B P P_{-}$and $C L B P_{-} C$ are fused to construct a $C L \bar{B} P$ operator named $S M C$ with Eq. (7).

$$
\begin{aligned}
& S M C=k_{s} C L B P_{-} S+k_{m} C L B P_{-} M+k_{c} C L B P_{-} C \\
& k_{s}+k_{m}+k_{c}=1
\end{aligned}
$$

where $k_{s}, k_{m}$ and $k_{c}$ are weight coefficients.

The $S M C$ operator describes the textural features of each pixel in the image.

Images $\mathrm{A}$ and $\mathrm{B}$ are assumed to be input original images for fusion, and $\mathrm{F}$ is the fusion result. Then, the relative textural feature $T_{F}^{A B}(p)$ of original images $\mathrm{A}$ and $\mathrm{B}$ and fusion image $\mathrm{F}$ is defined as follows:

$T_{F}^{A B}(p)= \begin{cases}\frac{S M C_{F}(p)}{S M C_{A}(p)}, & S M C_{A}(p) \geq S M C_{B}(p) \\ \frac{S M C_{F}(p)}{S M C_{B}(p)}, & S M C_{A}(p)<S M C_{B}(p)\end{cases}$

and $0 \leq T_{F}^{A B}(p) \leq 1$. A large $T_{F}^{A B}(p)$ value results in additional textural details acquired by the pixel in the fusion image from the original images.

Fusion image quality $Q_{F}^{A B}$ is then defined as:

$Q_{F}^{A B}=\frac{\sum_{m=0}^{M-1} \sum_{n=0}^{N-1} \gamma_{A B}(m, n) T_{F}^{A B}(m, n)}{\sum_{m=0}^{M-1} \sum_{n=0}^{N-1} \gamma_{A B}(m, n)}$

where $\gamma_{A B}(m, n)$ expresses the textural weight defined as follows:

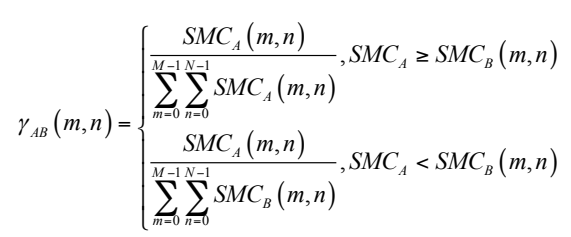

and $0 \leq Q_{F}^{A B} \leq 1$. A large $Q_{F}^{A B}$ value leads to additional textural details acquired in the fusion image from the original images and an improved fusion quality.

The CLBP-based fusion image quality evaluation method can be divided into the following four steps:

Step 1: $S M C_{X}(m, n)$ is calculated in accordance with Eq. (7), where $X=A, B$, and $F$;

Step 2: $T_{F}^{A B}(m, n)$ is calculated in accordance with Eq. (9);

Step 3: $\gamma_{A B}(m, n)$ is calculated in accordance with Eq. (11);

Step 4: Fusion image quality is obtained by using the results in Steps 2 and 3 in accordance with Eq. (10).

\section{Results analysis and discussion}

Experimental measurement and performance comparison of various multi-focus image fusion algorithms were conducted using Xydeas[25], FMI, and the proposed method in this study under MATLAB platform to verify the evaluation effect of the proposed fusion image quality evaluation method; these algorithms include contrast algorithm [26], variance algorithm [27], SF algorithm [28], image segmentation-based algorithm [29], and Gabor filter bankbased algorithm [30].

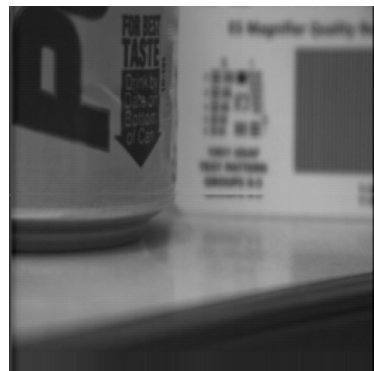

(a)

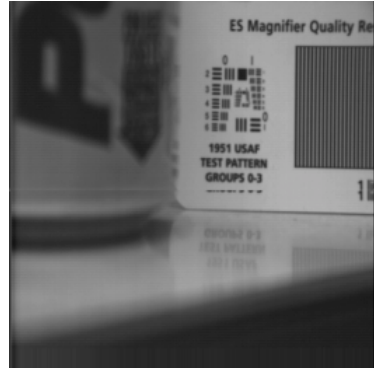

(b) 


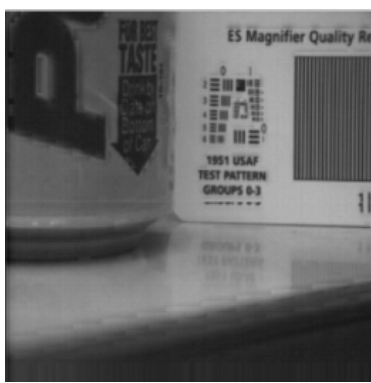

(c)

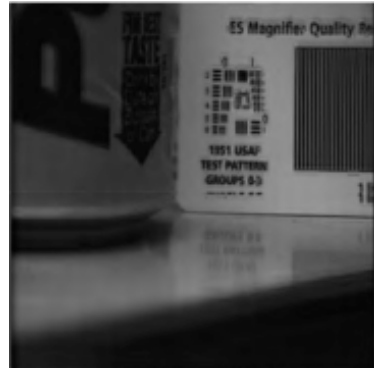

(e)

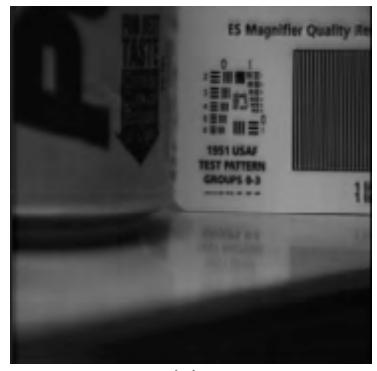

(g)

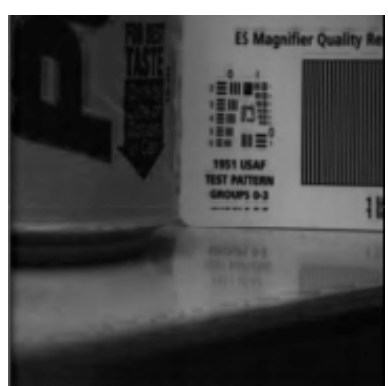

(d)

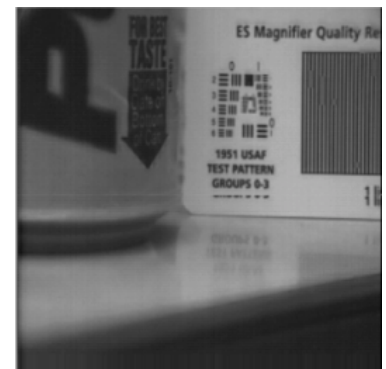

(f)
Fig. 1. Different fusion algorithms for the same original images (a) Original image A. (b) Original image B. (c) Segmentation-based. (d) SF. (e) Contrast. (f) Gabor filter bank-based. (g) Variance

The fusion results of the different fusion algorithms for the same original images are illustrated in Fig. 1. The results of the subjective analysis show the evident appearance of block effect in Figs. 1 (c) and (d) and the poor fusion effect. Through a local comparison, the effect in Fig. 1 (c) is clearly the poorest, and Figs. 1 (e), (f), and (g) exert favorable fusion effects with an acceptable definition. Based on further local comparison among the three figures, Fig. 1 (g) demonstrates the optimum effect. An artifact that results in the poorest fusion effect is depicted in Fig. 1 (e). Tables 1 and 2 present the comparison of fusion effects using objective indexes. Xydeas and FMI can evaluate the fusion effect to a certain degree, but the evaluation effect is inconsistent with the human subjective feeling under a few circumstances. The proposed algorithm considered the characteristics of the original images for fusion to objectively measure the fusion effect, which is relatively consistent with the human subjective feeling.

Table 1. Fusion result evaluation of the same original images

\begin{tabular}{l|l|l|l}
\hline $\begin{array}{l}\text { Images to be } \\
\text { evaluated }\end{array}$ & Xydeas & FMI & $\begin{array}{l}\text { Proposed method } \\
\left(k_{s}=0.3, k_{m}=0.5,\right. \\
\left.k_{c}=0.2\right)\end{array}$ \\
\hline Fig. 1. (c) & 0.7590 & 0.8416 & 0.8523 \\
Fig. 1. (d) & 0.7635 & 0.7625 & 0.8614 \\
Fig. 1. (e) & 0.5409 & 0.8891 & 0.9012 \\
Fig. 1. (f) & 0.6546 & 0.9094 & 0.9158 \\
Fig. 1. (g) & 0.6147 & 0.9092 & 0.9276 \\
\hline
\end{tabular}

Table 2. Consistency statistics of the evaluation result in Table. 2 with the subjective feeling

\begin{tabular}{|c|c|c|c|}
\hline $\begin{array}{l}\text { Images to be } \\
\text { evaluated }\end{array}$ & Xydeas & FMI & $\begin{array}{l}\text { Proposed method } \\
\left(k_{s}=0.3, k_{m}=0.5,\right. \\
\left.k_{c}=0.2\right)\end{array}$ \\
\hline Fig. 1. (c) & Consistent & Consistent & Consistent \\
\hline Fig. 1. (d) & Inconsistent & Consistent & Consistent \\
\hline Fig. 1. (e) & Consistent & Inconsistent & Consistent \\
\hline Fig. 1. (f) & Consistent & Inconsistent & Consistent \\
\hline Fig. 1. (g) & Inconsistent & Inconsistent & Inconsistent \\
\hline $\begin{array}{l}\text { Consistency } \\
\text { proportion }\end{array}$ & $60 \%$ & $40 \%$ & $80 \%$ \\
\hline
\end{tabular}

The fusion effects of contrast algorithm for different original images are displayed in Fig. 2, Tables 3 and 4 summarizes the evaluated fusion effects using objective indexes. Compared with Xydeas and FMI, evaluating the fusion effect through the proposed algorithm in this study is relatively consistent with subjective feeling.

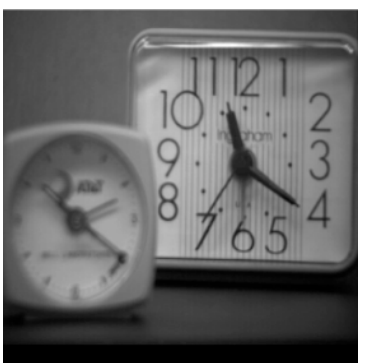

(a)

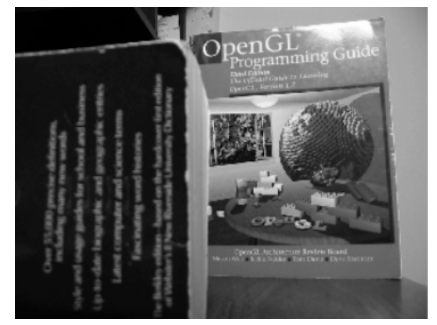

(c)

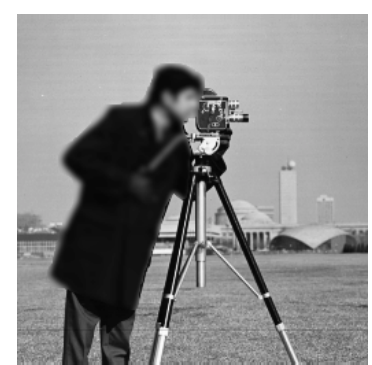

(e)

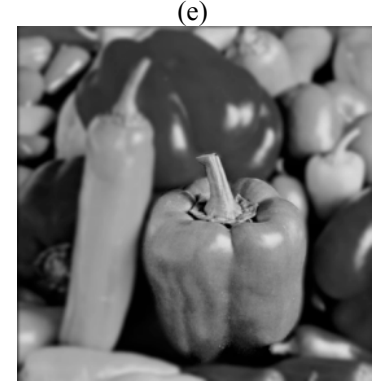

(g)

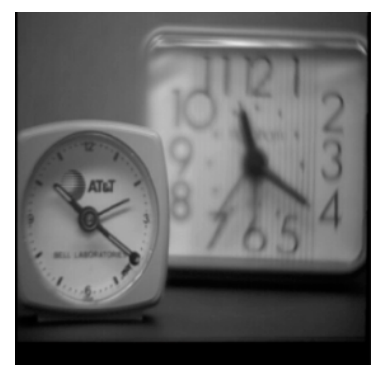

(b)

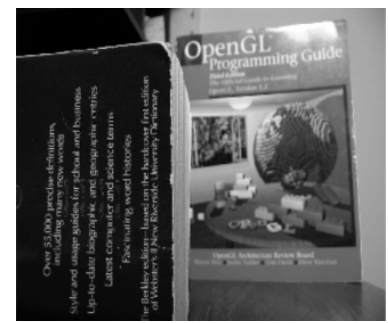

(d)

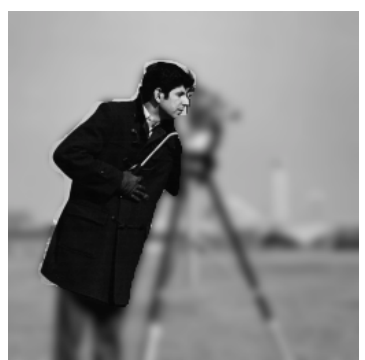

(f)

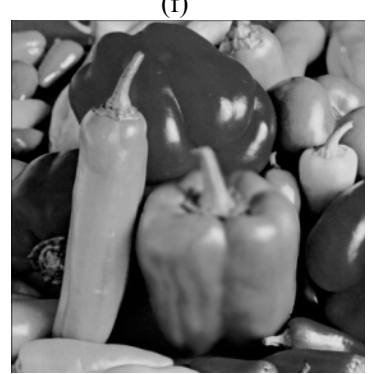

(h) 


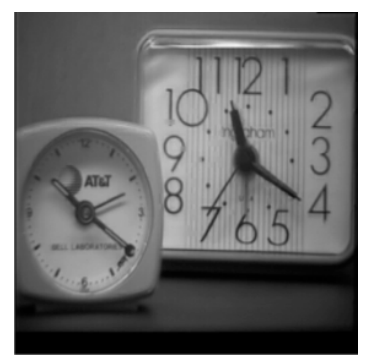

(i)

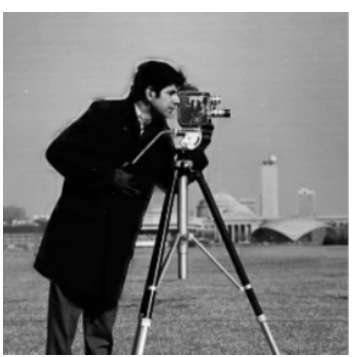

(k)

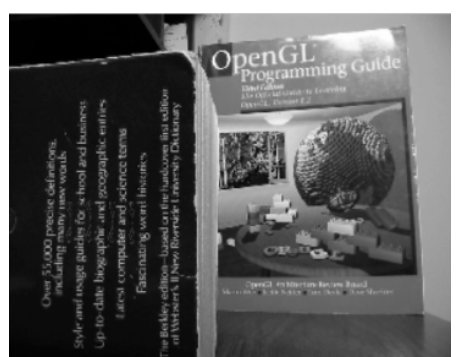

(j)

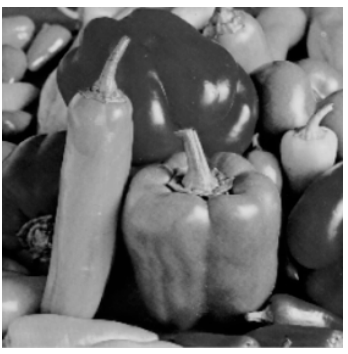

(1)
Fig. 2. Fusion images of the contrast algorithm for the different original images

(a) Original image A. (b) Original image B. (c) Original image C. (d) Original image D. (e) Original image E. (f) Original image F. (g) Original image G. (h) Original image H. (i) Fusion image Fa. (j) Fusion image Fb. (k) Fusion image Fc. (1) Fusion image Fd.

Table 3. Evaluation of the image fusion of the contrast algorithm

\begin{tabular}{l|l|l|l}
\hline $\begin{array}{l}\text { Images to be } \\
\text { evaluated }\end{array}$ & Xydeas & FMI & $\begin{array}{l}\text { Algorithm proposed } \\
\left(k_{s}=\mathbf{0 . 2 9}, k_{m}=\mathbf{0 . 5 2},\right. \\
\left.k_{c}=\mathbf{0 . 2 1}\right)\end{array}$ \\
\hline Fig. 2. (i) & 0.6587 & 0.8958 & 0.8796 \\
Fig. 2. (j) & 0.6971 & 0.9501 & 0.9015 \\
Fig. 2. (k) & 0.5896 & 0.9635 & 0.9249 \\
Fig. 2. (1) & 0.6012 & 0.8246 & 0.9301 \\
\hline
\end{tabular}

Table 4. Consistency statistics of the evaluation result in Table. 3 with the subjective feeling

\begin{tabular}{l|l|l|l}
\hline $\begin{array}{l}\text { Images to be } \\
\text { evaluated }\end{array}$ & Xydeas & FMI & $\begin{array}{l}\text { Algorithm proposed } \\
\left(k_{s}=\mathbf{0 . 2 9}, k_{m}=\mathbf{0 . 5 2},\right. \\
\left.k_{c}=\mathbf{0 . 2 1}\right)\end{array}$ \\
\hline Fig. 2. (i) & Consistent & Inconsistent & Consistent \\
\hline Fig. 2. (j) & Consistent & Inconsistent & $\begin{array}{l}\text { Consistent } \\
\text { Fig. 2. (k) }\end{array}$ \\
Inconsistent & $\begin{array}{l}\text { Consistent } \\
\text { Fig. 2. (1) }\end{array}$ & Inconsistent \\
& & Inconsistent & Inconsistent
\end{tabular}

\begin{tabular}{l|l|l|l} 
Consistency & $50 \%$ & $25 \%$ & $75 \%$
\end{tabular} proportion

\section{Conclusions}

Images of a medium complex texture were used as an example, and the CLBP was used in this study to construct the SMC operator to reveal the influence of original images with different quality for fusion image quality. The CLBP texture detection of the original images for fusion was conducted; the qualities of the parts in the original images were compared, and an objective evaluation method of fusion image was proposed. The following conclusions could be drawn:

(1) The quality of the image for fusion significantly influence the fusion result. The region with favorable quality where the original image is located significantly influences the fusion result. Moreover, the fusion result is improved when the proportion of the region in the fusion result is large.

(2) The image quality can be measured by describing the local texture using the CLBP algorithm. The combination of $C L B P_{-} S, C L B P_{-} M$, and $C L B P_{-} C$ can realize an elaborate description of the texture.

(3) The $C L B P M$ operator exerts the main effect when image texture is described by combining the $C L B P \_S$, $C L B P_{-} M$, and $C L B P_{-} C$ operators.

In this study, comparing image qualities for fusion is added to evaluate the fusion effects, which could accurately reflect the fusion image quality and provide a reference for selecting fusion algorithms. However, only textural features are considered in describing the fusion image quality. Therefore, additional influencing factors should be comprehensively considered to measure the fusion image quality in future studies to acquire an accurate evaluation of the fusion image quality.

This is an Open Access article distributed under the terms of the Creative Commons Attribution License

\section{References}

1. Ahmet Nusret Toprak, Veysel Aslantaş, "Spatial domain multi-focus image fusion". In: 2017 25th Signal Processing and Communications Applications Conference (SIU), Antalya, Turkey: IEEE, 2017, pp.1-4.

2. Veysel Aslantas, Emre Bendes, Rifat Kurban et al., "New optimised region-based multi-scale image fusion method for thermal and visible images". IET Image Processing, 8(5), 2014, pp.289-299.

3. Andreas Ellmauthaler, Carla L. Pagliari, Eduardo A. B. da Silva, "Multiscale image fusion using the undecimated wavelet transform with spectral factorization and nonorthogonal filter banks". IEEE Transactions on Image Processing, 22(3), 2013, pp.1005-1017.

4. Vikrant Bhateja, Himanshi Patel, Abhinav Krishn, et al., "Multimodal medical image sensor fusion framework using cascade of wavelet and contourlet transform domains". IEEE Sensors Journal, 15(12), 2015, pp.6783-6790.

5. Wang Z, Bovik A C, Sheikh H R, Simoncelli E P., "Image quality assessment: from error visibility to structural similarity". IEEE Transactions on Image Processing, 13(4), 2004, pp. 600-612.

6. Haghighat M, Razian M A., "Fast-FMI: non-reference image fusion metric". In: IEEE 8th International Conference on Application of Information and Communication Technologies, Astana, Kazakhstan: IEEE, 2014, pp.1-3.
7. PANG Hao-chen, ZHU Ming, GUO Li-qiang, "Objective color image fusion performance index". Optics and Precision Engineering, 21(9), 2013, pp.2348-2353.

8. ZHANG Yu-zhen, SUN Jia-song, CHEN Qian, et al., "Image fusion quality assessment method based on phase congruency and structural similarity". Acta Armamentarii, 34(12), 2013, pp.15141520 .

9. Luo Xiao-Qing, Wu Xiao-jun. "An evaluation method of image fusion based on region similarity". Acta Electronica Sinica, 38(5), 2010, pp.1152-1155.

10. ZHOU Pu-cheng, WANG Feng, CUI Xun-xue, et al., "Performance evaluation approach to image fusion based on ordering approximate to ideal solution". Systems Engineering and Electronics, 33(3), 2011, pp.681-684.

11. Zhu Yahui, Peng Guohua, Zheng Yushan, "Synthesized performance of infrared and visible image fusion based on kernel Fisher discriminant analysis( KFDA)". Journal of Northwestern Polytechnical University, 31(5), 2013, pp.770-773.

12. Wang Xiao-wen, Zhao Zong-gui, Tang Lei, "Novel quality metric for infrared and visible image fusion". Systems Engineering and Electronics, 34(5), 2012, pp.871-875. 
13. Wu Ruijuan, He Xiufeng, Wang Jing, "Quality assessment of fusion of ZY-3 multispectral and panchromatic images for coastal wetland areas". High Technology Letters, 25(2), 2015, pp.157-162.

14. Rania Hassen, Zhou Wang, Magdy M. A. Salama, "Objective quality assessment for multi-exposure multifocus image fusion". IEEE Transactions on Image Processing, 24(9), 2015, pp.27122724.

15. Vladimir Petrović; Boban Bondžulić; Rade Pavlović, "Study of objective evaluation of natural colour image fusion". In: IEEE 15th International Conference on Information Fusion, Singapore, Singapore: IEEE, 2012, pp.2523-2530.

16. Zaid Omar, Tania Stathaki, "GLCM-based metric for image fusion assessment". In: IEEE 15th International Conference on Information Fusion, Singapore, Singapore: IEEE, 2012, pp. 376381.

17. M.Hossny, S. Nahavandi, D.Creighton, et al., "Image fusion performance metric based on mutual information and entropy driven quadtree decomposition". Electronics Letters, 46(18), 2010, pp.1266-1268.

18. Nedeljko Cvejic, Tapio Seppänen, Simon J. Godsill, “A nonreference image fusion metric based on the regional importance measure". IEEE Journal of Selected Topics in Signal Processing, 3(2), 2009, pp.212-221.

19. N.Cvejic, C.N.Canagarajah, D.R.Bull, "Image fusion metric based on mutual information and Tsallis entropy". Electronics Letters, 42(11), 2006, pp.626-627.

20. Erik Blasch, Xiaokun li, Genshe chen, et al., "Image quality assessment for performance evaluation of image fusion". In: 2008 11th International Conference on Information Fusion, Cologne, Germany: IEEE, 2008, pp.1-6.
21. Ojala T, Pietika"inen M, Harwood D, "A comparative study of texture measures with classification based on featured distributions". Pattern Recognition, 29(1), 1996, pp.51-59.

22. Guo $\mathrm{Z} \mathrm{H}$, Zhang L, Zhang $\mathrm{D}$, "Rotation invariant texture classification using LBP variance (LBPV) with global matching”. Pattern Recognition, 43(3), 2010, pp.706-719.

23. Jing song $\mathrm{Xu}$, Qiang $\mathrm{Wu}$, "Fast and accurate human detection using a cascade of boosted MS-LBP features". IEEE Signal Processing Letters, 10, 2012, pp.676-679.

24. Guo Z., Zhang L., Zhang D, "A completed modeling of local binary pattern operator for texture classification". IEEE Transactions on Image Processing, 6(19), 2010, pp.1657-1663.

25. Xydeas C S, Petrovic V., "Objective image fusion performance measure". Electronics Letters, 36(4), 2000, pp. 308-309.

26. Tang J, "A contrast based image fusion technique in the DCT domain”. Digit Signal Process, 14(3), 2004, pp.218-226.

27. Haghighat M B A, Aghagolzadeh A, Seyedarabi H, "Multi-focus image fusion for visual sensor networks in DCT domain". Computers and Electrical Engineering, 37(5), 2011, pp.789-797.

28. Cao L, Jin L, Tao H, "Multi-focus image fusion based on spatial frequency in discrete cosine transform domain". IEEE Signal Processing Letters, 22(2), 2015, pp.220-224.

29. Li S, Yang B, "Multifocus image fusion using region segmentation and spatial frequency". Image and Vision Computing, 26(7), 2008, pp. 971-979.

30. Li X J, Wang M H, "Research of multi-focus image fusion algorithm based on Gabor filter bank'. In: Proceedings of 12th International Conference on Signal Processing, Hangzhou, China: IEEE, 2014, pp.693-697. 